\title{
LA BELIGERANCIA
}

\section{como camino hacia la SUBJETIVIDAD INTERNACIONAL de LOS PUEBLOS}

Estudiantes de Décimo Semestre de Derecho.

Universidad Surcolombiana.
Dorian Andrés Peralta

Luz Stella Loibo Ibarka

\section{Resumen}

El estudio de la beligerancia en el Derècho Internacional Humanitario, en la modernidad, requiere de una armonización de la semántica y desarrollo conceptual de un tema controvertido para la doctrina, y que, generalmente, aparece en la bibliografía con un tratamiento restringido por la mayoría de los publicistas de la materia, con la existencia de cinco corrientes teóricas que analizan la beligerancia en la legislación internacional. A partir de la conceptualización, se analizan las normas internas presentes en el contexto nacional establecidas como "reglas de guerra" que intentan delimitar la acción violenta de los actores armados al margen de la ley y procuran el respeto a los derechos humanos para los sujetos del DIH en medio del conflicto interno.

La caracterización del fenómeno bélico en Colombia, permite inferir que los sujetos del Derecho Internacional Humanitario no solamente se atribuye a los entes abstractos entendidos en este caso como Estado con todos sus componentes (territorio, la organización, población, etc.) y las organizaciones insurgentes; es también reconocer el hecho histórico que otros sujetos del DIH como Iglesia, Organizaciones No Gubernamentales, Activistas Sociales, también hacen parte del tejido social que participan como factores de mediación en los procesos de paz o de atención a la comunidad para apoyarlos en casos específicos de indefensión ante acciones de carácter militar.

La Subjetividad Internacional de los Pueblos es el concepto que más puede aproximarse, desde las doctrinas internacionales, sobre la identificación del sujeto partícipe del reconocimiento del DIH en el país. Al respecto, el profesor Manuel Díaz de Velasco, de la

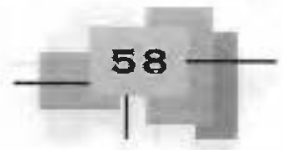


${ }^{1}$ El profesor Manuel Diaq de Velasco en su obra, Instituciones de Derecho Intemacional Público. ED. Colectivo de Abogados José Alvear Restrepo, 2.000 .

2 Estudio sobre Derecbo intemacional Humanitario, serie de texto de Gran $D$ ivulgación, Defensoria del Pueblo, Texto $N^{\circ} 11,1.996$.
Universidad del Rosario, señala que “...uno de los problemas más arduos y todavía más complejos del Derecho Internacional es el llegar a una delimitación acorde para individualizar el mecanismo por el que se ha llegado o se puede llegar a ser sujeto del ordenamiento internacional"

En este sentido, una de las corrientes teóricas más aceptadas es que los sujetos del DIH están inmersos en el conflicto armado; entre ellos se consideran a la Sociedad Civil, los Grupos Insurgentes (FARC, ELN, EPL, Paramilitares) y la Fuerza Pública. El jurista Díaz de Velasco argumenta que, sin haber agotado toda la casuística doctrinal, cabe señalar la posición que sostiene la existencia de una norma general respecto a la subjetividad aplicada a la mayoría de los sujetos y unas - normas especiales aplicables a los casos particulares.

En cuanto a las formas de atribuirse la subjetividad internacional, el profesor Díaz de Velasco, considera que "... existe una norma sobre la base de la cuál se atribuye ipso iure la subjetividad internacional a aquellas entidades en las que concurran ..." ${ }^{2}$. Los tratados internacionales de Ginebra, La Haya y el Pacto de Costa Rica, determinan esta condición que es ratificada por los Estados Nacionales, convirtiéndola en leyes de imperativo cumplimiento en los países que se obligan a cumplir dichos tratados.

Pero existen otras tendencias doctrinarias que conllevan al desarrollo de los sujetos del
Derecho Internacional, las cuales se determinan de la siguiente manera:

1. Aquella autores que considera como sujetos del derecho Internacional solamente a los Estados $y$, por consiguiente, que el DIH regula la relación entre los mismos.

2. Otra posición que considera que la subjetividad internacional surge como consecuencia de un acto jurídico de reconocimiento emanado de cada uno de los sujetos de DIH preexistente.

3. Una tercera posición frente al problema, es aquella que considera que existe, dentro del ordenamiento Jurídico Internacional, una norma única que atribuye la subjetividad internacional a todos aquellos que se encuentran en una determinada situación jurídica (Balladore - Pallieri).

4. Frente a ésta última se alza la del profesor Ago, y otros italianos como Ziccardi y Venturini, que niegan la existencia de dicha norma única y sostięnen que la subjetividad debe ser determinada por la ciencia jurídica a través de los datos que nos proporcione el estudio de las distintas normas internacionales y por los caracteres propios de sus destinatarios. Supone llegar a una valoración en concreto, caso por caso.

Carácterhistóricoy conceptualde la subjetividad internacional de lospueblos

Desde 1945, estamos asistiendo a un proceso de transformación profunda del Derecho Internacional. Es el

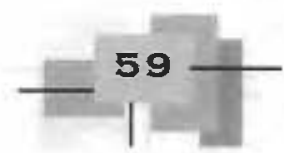




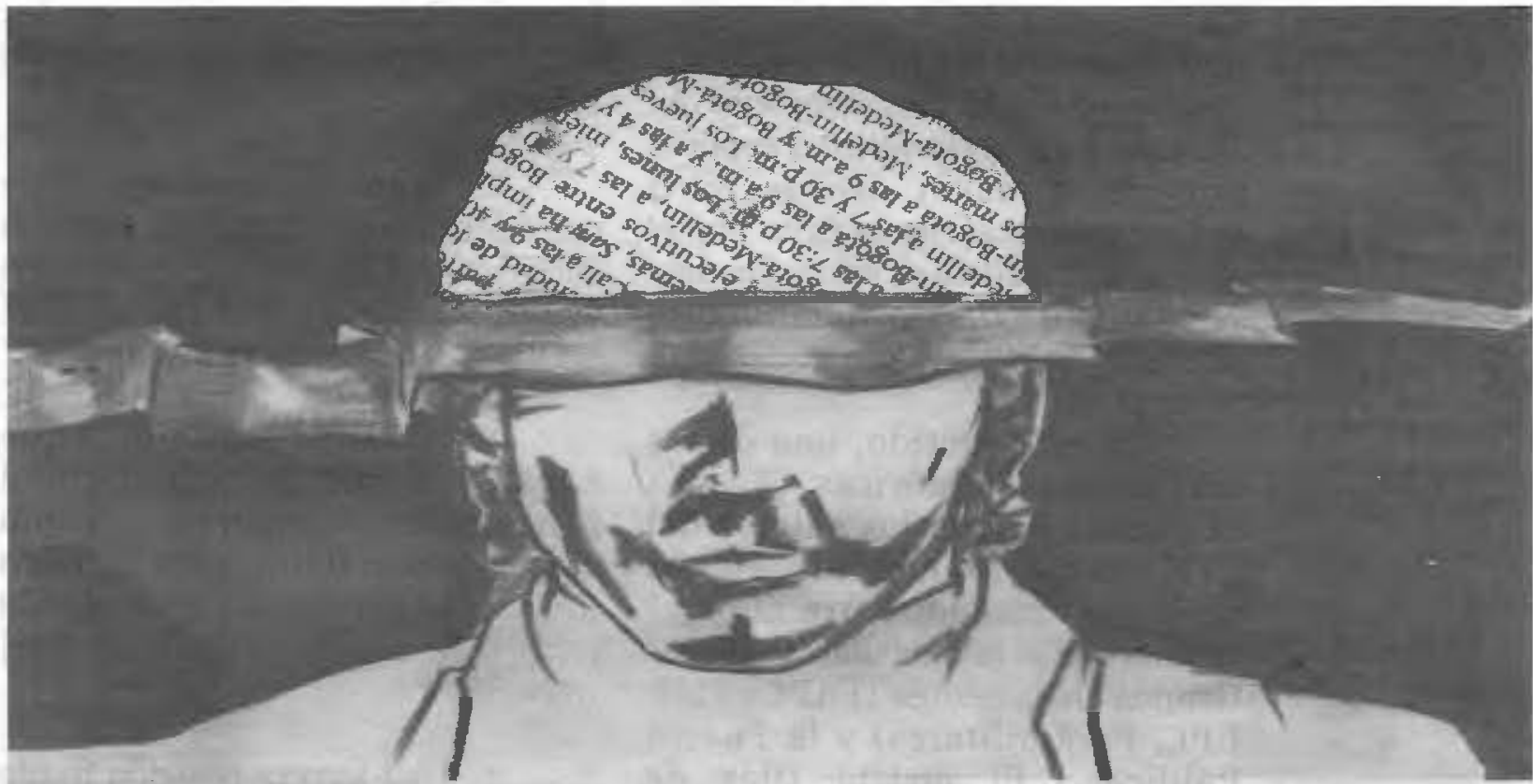

tránsito del derecho Internacional Clásico al derecho Internacional Contemporáneo, caracterizado :- por su contenido humanista y social, âdemás por la nueva función de procurar el desarrollo integral de los individuos y los pueblos sin excepción alguna.

Resulta necesario_aclarar que la situación de los pueblos en el Derecho Internacional no es la misma que cuando se los consideraba como simple objeto del ordenamiento, susceptibles de dominación extranjera, sino que se han convertido en titulares de muy importantes derechos; y entre estos derechos brilla con luz propia el de la libre determinación. El derecho a la libre determinación es el principal derecho de los prieblos, pero ello no significa que en el actual ordenamiento internacional sea éste el único derecho que poseen.

En otros ámbítos, también se reconocen derechos a los pueblos: derecho a expresar su voluntad en cuanto ésta es soporte de la soberanía; derecho a la supervivencia del grupo; derechos económicos y derechos a beneficios del ius in bello (Derecho de la Guerra). Los pueblos que recurren a la fuerza armada en su intento de alcanzar la libre determinación tienen también el derecho de beneficios de las normas del ius in bello.

En casos especiales, el grupo social (movimientos insurgentes) que se ha sublevado llega a controlar una parte del territorio del Estado y a establecer sobre el mismo una cierta organización. La existencia de tal situación, que se conoce técnicamente como beligerancia, puede ser reconocida tanto por el propio Gobierno del Estado donde acontece, como por terceros Estados y tiene una serie de consecuencias importantes. La principal, es la de aplicar a la contienda civil los derechos y obligaciones derivados del derecho de guerra y de neutralidad. La comunidad rebelde posee ciertos derechos y obligaciones directamente emanados del orden jurídico internacional, si bien se trata de un sujeto de carácter provisional, pues la situación de hecho sobre la que se funda la subjetividad de los beligerantes está destinada a desaparecer.

El status que se les reconoce desaparece con la terminación de la guerra civil, cuando la insurrección es aplastada o, finalmente, si llega a controlar todo el

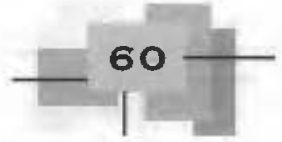


territorio, transformándose en éste último caso de Gobierno de facto local a Gobierno de facto general.

\section{Insurrección}

La Insurrección, en el contexto del Derecho Internacional, constituye un grupo de personas que se levanta en armas contra el gobierno de su propio Estado, controlando algunas plazas para imponer un modelo jurídico, económico y social. Esta situación, que es materia del derecho interno del Estado en cuestión, implica un reconocimiento, en el sentido de que sus actos oficiales no se consideran en principio como actos bélicos. Es muy discutida en la doctrina la cuestión de saber si existe un deber de reconocer a los insurrectos cuando se verifican las condiciones antes mencionadas, es decir el dominio efectivo y exclusivo de una importante zona del territorio de un Estado por parte de los sublevados. El Estado legalmente reconocido en elámbito internacional no está obligado a suministrar los recursos necesarios para eł sostenimiento y desarrollo de la comunidad que esta bajo el área de influencia de los insurrectos.

En relación con terceros Estados, la insurrección puede implicar derechos o privilegios que ellos han acordado conceder a la parte rebelde. Éstos varían de un Estado y de una situación a otros, porque la insurrección no es una condición, que como la beligerancia origine derechos y deberes definidos. Siendo ello así, no es posible determinar de antemano los elementos de la reacción de los Estados extranjeros ante la insurrección. Puede variar, desde la mera abstención de tratar a los rebeldes como "hostes generis humani", a un grado de relaciones semejantes a las mantenidas con el gobierno constitucional.

\section{Beligerancia o Rebeldía}

Hay sedición o rebelión en Derecho Internacional cuando en un Estado una organización o grupo social rebelde domina de hecho una parte apreciable del territorio y logra afirmarse en su lucha contra el gobierno central. El gobierno rebelde, que a diferencia del gobierno general de hecho, es un gobierno de carácter local, puede ser reconocido como beligerante por el gobierno central del propio estado donde acontece la rebelión, como así también por terceros Estados.

En ambos casos, la sedición se considera entonces como una guerra en el sentido del Derecho Internacional, siéndole aplicables las reglas del derecho de la guerra y la neutralidad.

\section{Reconocimiento de la Beligerancia}

De la misma manera que ocurre en el reconocimiento de Estados, podemos afirmar que existen en este tema dos escuelas de pensamiento opuesto. Una,

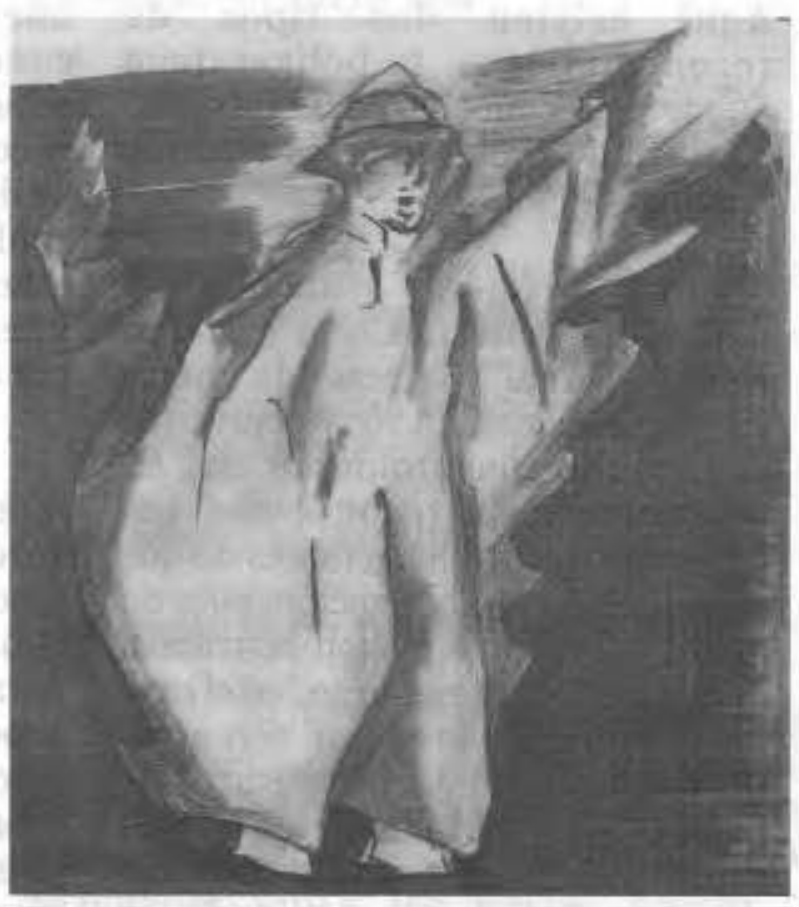


que considera el acto de reconocimiento como un otorgamiento o concesión de derechos, privilegios o de un status legal; la otra posición, meramente como una declaración de la existencia de ciertos hechos o un aviso de haberse enterado de ellos. Para la primera, un grupo rebelde carece de Derechos y no está sujeto a deberes en el Derecho Inter-nacional hasta que es reconocido. Para la segunda, la existencia de partes beligerantes es un hecho del cual se derivan los derechos y los deberes de los beligerantes y de los neutrales.

Si la sublevación se prolonga, puede convertirse en una guerra civil. La guerra civil existe, según el tratadista chileno Podestá Costa, desde el momento que se reconoce a los sublevados como beligerantes; desde ese momento son sujetos de derecho internacional, y las hostilidades'son regidas por el derecho internacional de la guerra. Aquí existen dos tîpos de reconocimiento: la beligerancia puede ser reconocida por el gobierno constituido o bien por los terceros Estados.

La primera situación la denomina Podésta Costa "Guerra Civil Nacional"3; la segunda guerra civil internacional. A veces ocurre que el gobierno constituido no está en condiciones de reconocer a sus adversarios como beligerantes sin que ello le genere una obligación, pues no existe ninguna condición restrictiva para hacerlo. Sin embargo, en el caso de reconocimiento por terceros, un reconocimiento prematuro, no fundado, constituiría una intervención en asuntos internos de otros Estados, y por ende un grave delito internacional.
Por lo tanto para que un tercer Estado pueda reconocer a los sublevados como beligerantes, esto tienen que responder a ciertas condiciones subjetivas: a) perseguir objetivos políticos, b) tener dirección política, c) disponer de fuerzas armadas disciplinadas, d) controlar un territorio, e) observar el derecho internacional de guerra. Se necesita además cierta relación entre ese tercer Estado y los sublevados. El, Estado que reconoce a los sublevados tiene que encontrarse en una situación tal que no pueda evitar el reconocimiento.

\section{Efectos del reconocimiento de beligerancia}

Dos importantes consecuencias jurídicas se derivan de este reconocimiento:

1) En lo sucesivo, se hace posible aplicar las reglas del derecho internacional sobre la manera de conducir las hostilidades, a las relaciones entre el gobierno legal reconocido y las autoridades beligerantes reconocidas. El conflicto civil se transforma en una guerra regida por el derecho internacional en todos sus aspectos, por ejemplo, la neutralidad.

2) La responsabilidad internacional por los actos de las autoridades beligerantes reconocidas se transfiere del gobierno legal a los beligerantes. Estas dos consecuencias se producen porque, por el control efectivo del gobierno insurgente sobre una parte del territorio y del pueblo perteneciente al Estado envuelto en la guerra civil, se forma una entidad que verdaderamente
${ }^{3}$ Halajcrute, Bohdan t.y Moya dominguez, Maria Teresa del $\mathrm{R}$. Derecho internacional Público. Editar. Buenos Aires. 1999, pág. 812/813.

${ }_{4}^{4}$ Akehurst, Michael. Introducción al $D e r e c b$ o internacional. Alianza Editorial. Madrid. 1973, p. 411.

5 Protocolo de Ginebra, agosto de 1949.

6 Ibid. Articulo $3^{\circ}$.

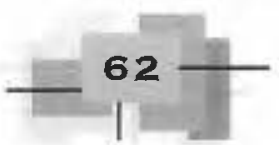


se parece a un Estado en el sentido del derecho internacional. Por otro lado, el gobierno establecido está impedido en el futuro de actuar arbitrariamente contra los rebeldes que han sido reconocidos.

A partir de ese momento se elevan del nivel local al internacional las relaciones entre las autoridades belig̈erantes reconocidas; el gobierno legal y los Estados que reconocen. Estos resultados legales son reconocidos y reafirmados en términos idénticos por las cuatro Convenciones de Ginebra de 1949, que en su Art. $3^{\circ}$ común, regulan respectivamente, la condición de los heridos y de los enfermos en campaña, la de los heridos, enfermos y náufragos en el mar, el tratamiento de los prisioneros de guerra y la protección de los civiles en tiempo de guerra.

El artículo $3^{\circ}$ de los Convenios de Ginebra de 1949, no ha conseguido una aplicación satisfactoria en la práctica. En caso de Guerra Civil, cada bando considera a los miembros del otro como traidores, y no es éste el terreno ideal para la aplicación del-derecho de la guerra. El artículo $3^{\circ}$, no prohíbe siquiera el fusilamiento de prisioneros de guerra por delito de traición, a condición de que la pena se imponga en un proceso razonable.

Además, las guerrillas y otras fuerzas irregulares juegan un papel muy importante en las guerras civiles, y esto dificulta el establecimiento de la distinción entre combatientes y población civil. $\quad \mathrm{Y}$ en el caso de "internacionalización" de la guerra civil por la partici-pación de fuerzas extranjeras, como por el ejemplo en la Guerra Civil Española de 1936/1939 (Legión Cóndor, Legión Italiana y Brigadas Internacionales), también la experiencia en las guerras de Yemen y Vietnam, indica que no aumentan las probabilidades de obediencia al
Derecho de la guerra. Sin embargo, el deseo de causar una impresión favorable sobre la opinión pública extranjera ha ejercido en ocasiones un efecto limitativo y ha influido en una cierta observancia del artículo $3^{\circ}$, en esta medida esto ha simbolizado "el temor a las represalias y a la persecución de los crímenes de guerra que también ha ejercido, en determinada casos, un efecto favorable"4.

Para finalizar, el hecho de la beligerancia, cuando va acompañado de las condiciones descritas, da a las partes de la contienda derechos y deberes internacionales otorgándoles la categoría de sujetos de derecho internacional. Como es fácil apreciar, la "comunidad beligerante" es un sujeto provisional, dado lo precario de la situación de hecho en que se funda, la beligerancia o gana el control del territorio y el gobierno, o es eliminada.

\section{Sujetos del conflicto armado en Colombia}

Los sujetos involucrados en el presente conflicto son:

- Los grupos armados: las Fuerzas Armadas Revolucio-narias de Colombia, el Ejército de Liberación Nacional, el Ejército Popular de Liberación y el Movimiento M-19, entre los más representativos.

- El Estado colombiano (Gobierno, Fuerzas Armadas).

- Grupos paramilitares.

\section{Tipología del conflicto colombiano}

En primer lugar, hay que puntualizar que el presente caso trata de un conflicto y no de una controversia, dado que aparte de los puntos en discusión entre las partes existe el componente de la violencia armada. 
Para determinar ante qué clase de conflicto nos hallamos, partimos de la existencia de dos tipos de conflicto que interesan para la aplicación del Derecho Internacional. Por un lado, el conflicto internacional, y por el otro, el conflicto interno. Entonces, debemos acudir a aquellos instrumentos que han - desarrollado el contenido y elementos de ambos conflictos.

El conflicto internacional se constituye cuando intervienen dos sujetos de derecho internacional y se aplica el derecho internacional. Esta consideración implica dos interrogantes de carácter jurídico: si los guerrilleros colombianos son sujetos de Derecho Internacional Humanitario o si se aplica en este conflicto normas de Derecho Internacional Humanitario.

En el primer cuestionamiento, el reconocimiento de la existencia del sujeto de derecho internacional es declarativo; sin embargo, a efectos de que pueda relacionarse con otros sujetos de derecho internacional en el ámbito internacional, es necesario que estos lo reconozcan cōmo tal.

A pesar de que un grupo en armas cumpla con los requisitos se adquiere la calidad de sujeto de derecho internacional. Adicionalmente, se infiere que los "grupos beligerantes" son los sujetos de derecho internacional cuyas características son las más similares a los grupos guerrilleros de Colombia.

Los grupos beligerantes en Colombia asumen características de tal condición en la doctrina porque poseen una fuerza militar organizada, autoridad responsable de los actos (en general, casi la totalidad de las guerrillas colombianas cuentan con una fuerza militar organizada), con una posesión parcial y dominio de una zona del territorio nacional (se puede decir que sólo las FARC y el ELN tienen dominio sobre determinado territorio; las demás fuerzas actúan de manera dispersa).

En lo concerniente a la aplicación del Derecho Internacional Humanitario, el grupo armado debe comprometerse a hacerlo (sólo las FARC han declarado que se comprometen a cumplir las disposiciones del DHI, sin embargo, la constatación de dicho cumplimiento se obstaculiza por las diferencias radicales y por las situaciones bélicas que en combate se presentan entre las partes que afrontan al conflicto colombiano).

Es necesario resaltar en este punto que la calidad $\mathrm{de}^{\star}$ beligerante fue creada en Derecho Internacional como una salida para poder atribuir responsabilidad internacional a los grupos armados por sus acciones dentro del territorio de un Estado preexistente. Esta categoría fue recogida por el DIH para poder incluir dentro de su ámbito de protección, situaciones que por no tener como sujetos a dos estados escapaban a su ámbito de protección.

Bajo estos elementos, debe analizarse que sólo las FARC se podrían considerar sujeto de derecho internacional, más no los otros grupos levantados en armas, incluido los grupos paramilitares.

\section{Desarrollo Normativo de DIH:}

La evolución del Derecho Internacional Humanitario, a través de los cuatro Convenios de Ginebra, y los dos Protocolos Facultativos delimita algunas normas de aplicación con respecto al derecho de la guerra. Para esta rama del derecho existe un conflicto internacional, cuando este surge entre dos o varias Altas Partes Contratantes, es decir, Estados (Artículo 2 común a los cuatro convenios de Ginebra y el artículo $1^{\circ}$ del Primer Protocolo Adicional). Desde este punto de vista, los grupos guerrilleros no podrían ser parte de un conflicto internacional.

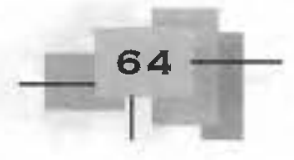




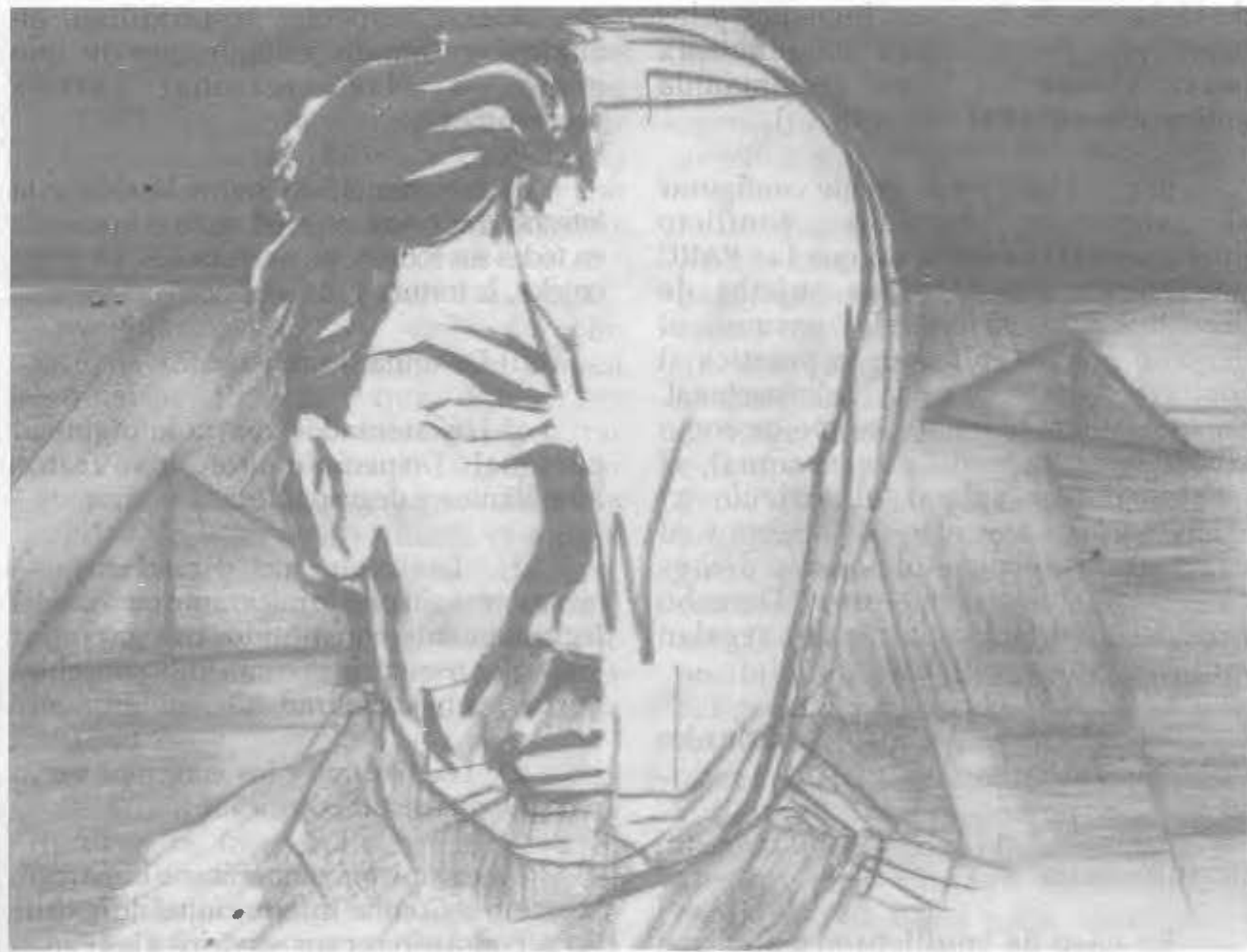

Esta posición se coñfirma con lo dispuesto por el Artículo 3 Común a estos convenios y el Segundo Protocolo Facultativo que desarrolla el concepto de conflicto armado no internacional a efectos de aplicar el Derecho Internacional Humanitario. Según el artículo 3 común, el conflicto armado no internacional es aquél que surge en el territorio de una de las Altas Partes Contratantes. El Protocolo II detalla más el concepto manifestando que.

\section{Artículo 1: ámbito de aplicación material}

1. “El presente Protocolo, que desarrolla y completa el artículo $3^{\circ}$ común a los Convenios de Ginebra del 12 de agosto de 1949, sin.modificar sus actuales condiciones de aplicación, se aplicará a todos los conflictos armados que no estén cubiertos por el artículo 1 del Protocolo adicional a los Convenios de Ginebra del 12 de agosto de 1949 relativo a la protección de las víctimas de los conflictos armados internacionales (Protocolo I) y que se desarrollen en el territorio de una Alta Parte contratante entre sus fuerzas armadas y fuerzas armadas disidentes o grupos armados organizados que, bajo la dirección de un mando responsable, ejerzan sobre una parte de dicho territorio un control tal que les permita realizar operaciones militares sostenidas y concertadas $y$ aplicar el presente Protocolo"s.

Respecto a este punto, de acuerdo a la situación colombiana, el conflicto se rige por las normas de derecho interno pues el Estado aplica las normas de su ordenamiento penal para juzgar a los guerrilleros implicados en actos

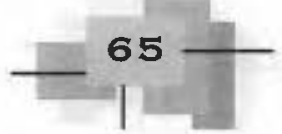


de violación de derechos humanos y los considera delincuentes comunes (a pesar de que las FARC reclaman la aplicación del DIH al conflicto).

- Por lo tanto no se puede configurar el supuesto de un conflicto internacional (a pesar de que las FARC pueden ser consideradas sujetos de derechos internacional) porque el derecho que se aplica en la práctica al conflicto no es el Derecho Internacional. Este conflicto se encuadra mejor como conflicto armado no internacional, al que se deben aplicar el artículo $3^{\circ}$ Común a los Convenios de Ginebra y Al Segundo Protocolo Adicional a dichos convenios, normas de Derecho Internacional Humanitario que regulan esta clase de conflictos.

En este sentido, el artículo $3^{\circ}$ común a los Convenios de Ginebra dice:

Artículo $3-$ Conflictos no
internacionales
En caso de conflicto armado que no sea de índole internacional y que surja en el territorio de una de las Altas Partes Contratantes, cada una de las Partes en conflicto tendrá la obligación de aplicar, como mínimo, las siguientes disposiciones:

1) Las personas que no participen directamente en las hostilidades, incluidos los miembros de las fuerzas armadas que hayan depuesto las armas y las personas puestas fuera de combate por enfermedad, herida, detención o por cualquier otra causa, serán, en todas las circunstancias, tratadas con humanidad, sin distinción alguna de índole desfavorable basada en la raza, el color, la religión o la creencia, el sexo, el nacimiento o la fortuna o cualquier otro criterio análogo.
A este respecto, se prohíben, en cualquier tiempo y lugar, por lo que atañe a las personas arriba mencionadas:

a) Los atentados contra la vida y la integridad corporal, especialmente el homicidio en todas sus formas, las mutilaciones, los tratos crueles, la tortura y los suplicios;

b) La toma de rehenes;

c) Los atentados contra la dignidad personal, especialmente los tratos humillantes y degradantes;

d) Las condenắs dictadas y las ejecuciones sin previo juicio ante un tribunal legítimamente constituido, con garantías judiciales reconocidas como indispensables por los pueblos civilizados.

2) Los heridos y los enfermos serán recogidos y asistidos.

Un organismo humanitario imparcial, tal como el Comité Internacional de la Cruz Roja, podrá ofrecer sus servicios a las Partes en conflicto.

Además, las Partes en conflicto harán lo posible por poner en vigor, mediante acuerdos especiales, la totalidad o parte de las otras disposiciones del presente Convenio.

La aplicación de las anteriores disposiciones no surtirá efectos sobre el estatuto jurídico de las Partes en conflicto"6

\section{Conclusiones}

Este artículo hace una diferenciación expresa del tipo de sujetos que intervienen en este tipo de conflictos, dado que a los Estados los llama "Parte" (resulta necesario evidenciar que el Estado en cuyo territorio se desarrolla el conflicto recibe ambas denominaciones). 
En general, el DIH amplía al máximo la interpretación de este tipo de categorías internacionales, y a veces inclusive las obvia, para poder cumplir con sus objetivos (la protección de las víctimas de los conflictos armados) con un mayor alcance y de manera más efectiva.

Sin embargo, en derecho internacional estas categorías tienen requisitos expresos que deben ser cumplidos. Por ello vamos a encontrar una contradicción entre el DIH que por doctrina señala que para tener la calidad de beligerantes basta el propio reconocimiento de dicha calidad, mientras que en derecho internacional se dice que sólo otros sujetos de derecho internacional preexistentes pueden reconocer y otorgar dicha calidad.

Adicionalmente, es importante resaltar la importancia que para el Estado colombiano tendrá el reconocimiento de la beligerancia de las FARC •dado que les atribuiríá responsabilidad internac̄ional por sus actos, sometería el conflicto a las reglas del DIH y tendríá la potestad de exigir la neutralidad de los otros Estados frente al conflicto, posibilidad que hoy en día pone en riesgo a toda la región al vislumbrarse una posible intervención estado-unidense a gran escala, con la finalidad aparente de fortalecer la lucha antidrogas, pero con la evidente ansia por eliminar a los grupos subversivos, tal como lo denuncia instituciones de la importancia de WOLA.

Enfatizar la relevancia de que Colombia reconozca la beligerancia de las FARC, dado que este reconocimiento pondría las reglas en claro entre las parte, situando el conflicto dentro de los límites de Colombia y evitando la intervención de potencias militares que podrían sacar de toda proporción este conflicto y llevarlo a extremos mucho más graves que los hasta ahora alcanzados, como es el caso de EE.UU., cuya práctica en estas situaciones es ampliamente conocida y repudiada.

Además, sostenemos que la ONU podría intervenir para salvaguardar la paz y seguridad internacional, previo reconocimiento de la calidad de interno de este conflicto, de modo que se asegure mayor imparcialidad a la hora de llegar a una solución definitiva de este conflicto armado que leva ya 35 años, para que futuros procesos de negociación se encuentren libres de los giros políticos que trae consigo el cambio de gobierno, que en la actualidad ve más factible reconoce la posibilidad de conceder el status de beligerancia a los paramilitares, para brindarles las garantías legales que ofrece el DIH y no continuar con el lento y dilatado proceso de San Vicente del Caguán que en cuatro años no dejo más que un país cansado de debatir en medio de las balas y los ataques a la población civil.

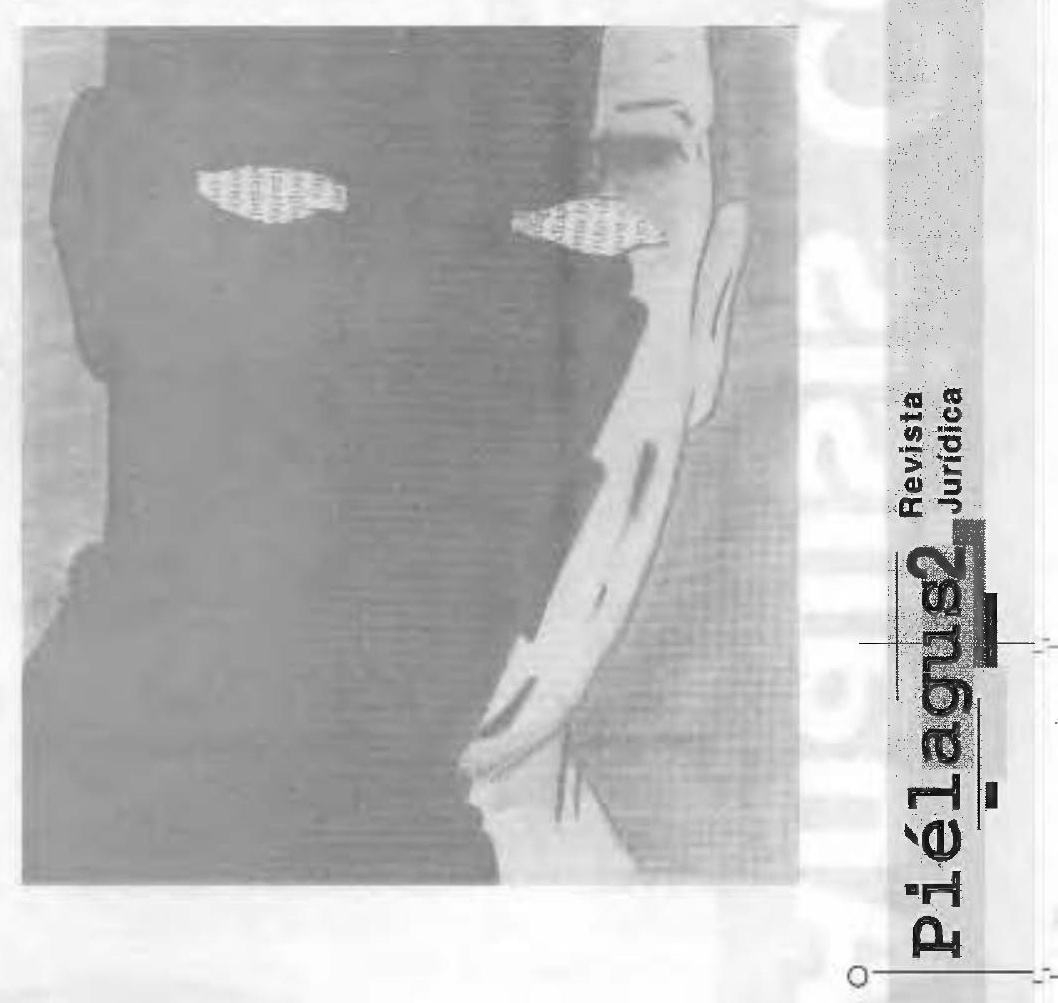

